\title{
Ultrastructure and histochemistry of rat myocardial capillary endothelial cells in response to diabetes and hypertension
}

\author{
Ludmila OKRUHLICOVA ${ }^{1, *}$, Narcis TRIBULOVA ${ }^{1}$, Peter WEISMANN ${ }^{2}$, Ruzena SOTNIKOVA ${ }^{3}$ \\ ${ }^{1}$ Institute for Heart Research, Slovak Academy of Sciences, 84005 Bratislava, Slovak Republic \\ ${ }^{2}$ Medical Faculty, Comenius University, Bratislava, Slovak Republic \\ ${ }^{3}$ Institute of Experimental Pharmacology, Slovak Academy of Sciences, Bratislava, Slovak Republic
}

\begin{abstract}
Insufficient growth and rarefaction of capillaries, followed by endothelial dysfunction may represent one of the most critical mechanisms involved in heart damage. In this study we examined histochemical and ultrastructural changes in myocardial capillary endothelium in two models of heart failure streptozotocin-induced diabetes mellitus (STZ) and NOdeficient hypertension in male Wistar rats. Diabetes was induced by a single i.v. dose of STZ ( $45 \mathrm{mg} / \mathrm{kg}$ ) and chronic 9week stage was analysed. To induce NO-deficient hypertension, animals were treated with inhibitor of NO synthase Lnitroarginine methylester (L-NAME) $(40 \mathrm{mg} / \mathrm{kg})$ for 4 weeks. Left ventricular tissue was processed for enzyme catalytic histochemistry of capillary alkaline phosphatase (AlPh), dipeptidyl peptidase IV (DPP IV), and endothelial NO synthase/NADPH-diaphorase (NOS) and for ultrastructural analysis. In diabetic and hypertensive rats, lower/absent AlPh and DPP IV activities were found in focal micro-areas. NOS activity was significantly reduced and persisted only locally. Quantitative evaluation demonstrated reduction of reaction product intensity of AlPh, DPP and NOS by $49.50 \%$, $74.36 \%, 20.05 \%$ in diabetic and $62.93 \%, 82.71 \%, 37.65 \%$ in hypertensive rats. Subcellular alterations of endothelial cells were found in heart of both groups suggesting injury of capillary function as well as compensatory processes. Endothelial injury was more significant in diabetic animals, in contrast the adaptation was more evident in hypertensive ones. Concluding: both STZ-induced diabetes- and NO-deficient hypertension-related cardiomyopathy were accompanied by similar features of structural remodelling of cardiac capillary network manifested as angiogenesis and angiopathy. The latter was however, predominant and may accelerate disappearance of capillary endothelium contributing to myocardial dysfunction.
\end{abstract}

Keywords: rat heart, diabetes, NO-deficiency, capillary, ultrastructure, histochemistry.

\section{INTRODUCTION}

The streptozotocin [STZ]-induced diabetes and NOdeficient hypertension are widely used experimental models for the investigation of heart failure [1-4]. Cardiac dysfunction in both models is consequence of different signal and metabolic derangement which continuously results in structural remodelling of cardiovascular system. The latter is manifested by myocardial hypertrophy and fibrosis as well as coronary artery wall hyperplasia [5-

\footnotetext{
*Correspondence: Ludmila OKRUHLICOVA

Institute for Heart Research, Slovak Academy of Sciences, Dubravska cesta 9, 84005 Bratislava 45, PO Box 104, Slovak Republic

Tel: + 4212547744 05; Fax: + 421254776637

E-mail: usrdokru@savba.sk
}

11] that consequently lead to the heart injury known as cardiomyopathy.

Evidence is rapidly accumulating that morphological alteration of the finest segments of vascular system capillaries - insufficient growth and rarefaction - may represent one of the most critical mechanisms involved in the heart failure [12-15]. Myocardial capillary wall consists of a continuous monolayer of endothelial cells which form a selective permeable barrier. Capillary wall is covered by pericytes that are multifunctional cells involved also in repair process [16]. Capillaries' major function is to promote exchange of oxygen nutrients and metabolic products between the blood and interstitial tissue. Endothelial cells secret a wide range of biologically active mediators which modulate many physiological activities of cardiovascular system [17]. Thus, functional and structural properties of 
capillaries predispose them to respond quickly to local changes in biological needs of the myocardium [18]. The endothelial cells are able to adapt to temporal and local requirements. However, their exposition to chronic pathophysiological stimuli or conditions can result in endothelial dysfunction which represents a critical moment in the remodelling processes of vascular wall, extracellular space and consequently the myocardium. These alterations do not occur simultaneously. They may progress due to endothelial structural and functional heterogeneity, intrinsic properties of endothelium as well as nature of the injury $[19,20]$.

We focused in this study on histochemical and ultramicroscopic examination of myocardial capillary network in two various animal models of heart failure STZ-induced diabetes and NO-deficient hypertension.

\section{MATERIALS AND METHODS}

The study and experimental protocol were approved and monitored by the State Veterinary and Food Authority of Slovak Republic Act No. 488/2002.

Male adult Wistar rats [250-300 g body weight] were divided into three groups: control, diabetic and hypertensive animals $[n=6$ per each group].

\section{Animal model of diabetes mellitus}

Experimental type I diabetes mellitus was induced by a single intravenous injection of STZ [45 mg/kg] [3]. STZ was dissolved in $0.1 \mathrm{~mol} / 1$ citrate buffer, $\mathrm{pH}$ 4.5. Four days after STZ administration all animals with plasma glucose level $>20 \mathrm{mmol} / \mathrm{l}$ were considered diabetic and included into the study.

The nine-week diabetes reduced heart rate by $27 \%$, the body weight and heart weight by $28 \%$ and $9 \%$, respectively. Relative heart weight [heart weight/body weight ratio] increased by $26 \%$, comparing with age-matched controls.

\section{Animal model of NO-deficient hypertension}

Hypertension was induced by application of L-nitroarginine methylester [L-NAME], the inhibitor of NO synthase, in a daily dose of $40 \mathrm{mg} / \mathrm{kg}$ in drinking water for $4 \mathrm{w}$ [2]. After that, there was a significant increase in systemic blood pressure by $30 \%$. Heart rate was reduced by $23 \%$, and relative left ventricle weight increase by $27 \%$, as compared to the control group.

At the end of experiments, the animals were killed by cervical dislocation, the heart was rapidly excised and processes for enzyme histochemical and electron microscopic examination.

\section{Catalytic enzyme histochemistry}

The tissue of the left ventricle was immediately frozen in liquid nitrogen followed by cutting into $10 \mu \mathrm{m}$ thick cryostat sections [Cryotome Shandon, Great Britain]. The activity of endothelial NADPH-diaphorase/NOS [NOS] [E.C.1.14.13.39.] [21], alkaline phosphatase [AlPh] [E.C.3.1.3.1.] - marker of arterial part of capillaries, and dipeptidyl peptidase IV [DPP IV] [E.C.3.4.15.4] marker of venular part of capillaries were performed on unfixed cryostat sections according to Lojda et al. [22]. The intensity of colour staining reflected the activity of detected enzymes and was quantified.

\section{Quantitative analysis}

The intensity of enzyme reactions in capillaries was measured using a light microscope [IMT-4, Olympus, Japan] with attached CCD camera [Jai 2040, Jai, Japan] and morphometric software [AnalySIS, Soft Imaging system, Germany]. Each of the measurements [control and experimental groups] was performed blindly, and under similar conditions of light, offset and magnification. Ten transmural cryostat slices of each heart stained for AlPh, DPP IV and NOS were analysed. Statistical analysis was performed using ANOVA.

\section{Transmission electron microscopy}

Ventricular tissue was cut into small pieces and fixed with $2.5 \%$ glutaraldehyde in $100 \mathrm{mmol} / \mathrm{l}$ cacodylate buffer [pH 7.4] for $3 \mathrm{~h}$. After washing, tissue was postfixed in $1 \% \mathrm{OsO}_{4}$, dehydrated via ethanol series, infiltrated by propylene oxide and embedded in Epon
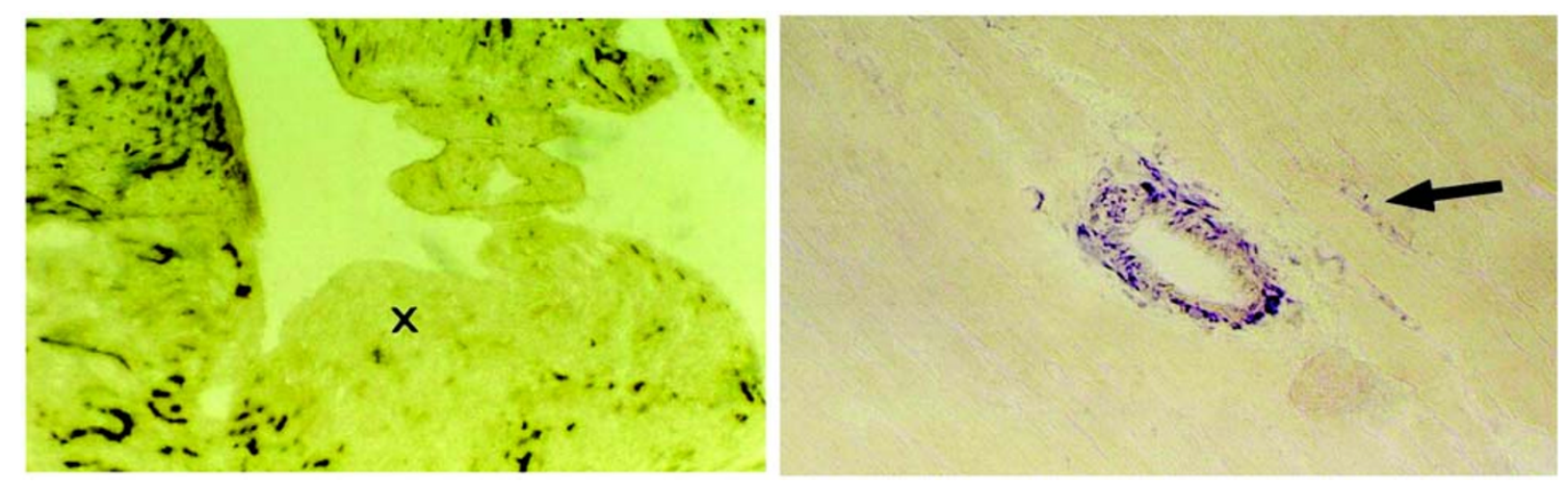

Fig. 1 Histochemical demonstration of capillary alkaline phosphatase in the myocardium of hypertensive rats (A) and NO synthase in the diabetic one (B). Alkaline phosphatase was heterogeneously decreased $(\times)$, while NO synthase persisted only locally (arrow). Magnification: A $\times 150 ; \mathbf{B} \times 150$. 
812. Ultrathin sections cut on ultramicrotome LKB Huxley [London, Great Britain] were counter-stained with uranyl acetate and lead citrate and examined in electron microscope Tesla 500 [Brno, Czech Republic].

\section{Cytochemical detection of NO synthase}

Small pieces of ventricular tissue of all experimental groups were fixed in $1 \%$ glutaraldehyde for $5 \mathrm{~min}$, washed and for enzyme activity detection incubated in medium according to Lojda et al. [22]. After post-fixation in $1 \% \mathrm{OsO}_{4}$ the tissue was routinely processed for transmission electron microscopy.

\section{RESULTS}

\section{Enzyme histochemistry}

Capillary enzyme activity of AlPh and DPP IV reactions were homogeneously distributed within the whole myocardial tissue in control group. AlPh was nonuniformly reduced/abolished mainly in endocardium [Fig. 1A], while DPP IV activity was almost without changes. Unlike NOS activity was significantly reduced and persisted only in focal micro-areas within the myocardial tissue of diabetic and hypertensive animals [Fig. 1B]. The quantification of enzyme activity [Fig. 2] demonstrated significant decrease in AlPh and NOS.

\section{NO synthase cytochemistry}

In parallel with histochemistry, capillary NOS activity was detected on the level of electron microscopy. Specific product of the enzyme reaction was demonstrated only in a part of capillary endothelial cells in diabetic and L-NAME-treated animals [Fig. 3]. Endothelial cells due to the enzyme reaction product were electron dense, while in other ones the enzyme activity was absent.

\section{Myocardial capillary endothelial cells ultrastructure}

Ultramicroscopic analysis of capillaries showed hetero-

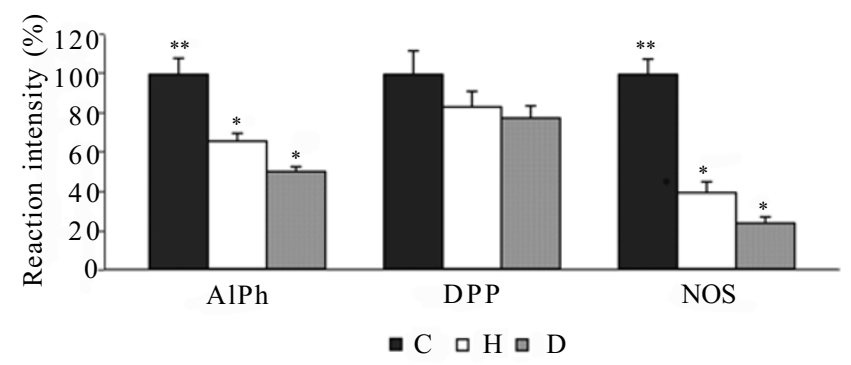

Fig. 2 Quantitative image analysis of intensity of capillary enzyme activities in the myocardium of control (C), hypertensive $(\mathrm{H})$ and diabetic (D) animals. AlPh - alkaline phosphatase, DPP - dipeptidyl peptidase IV, NOS - NO synthase coupled to NADPH-diaphorase. ${ }^{*} P<0.01$ (hypertension vs diabetes); ${ }^{* *} P<0.001$ (control vs hypertension and diabetes).

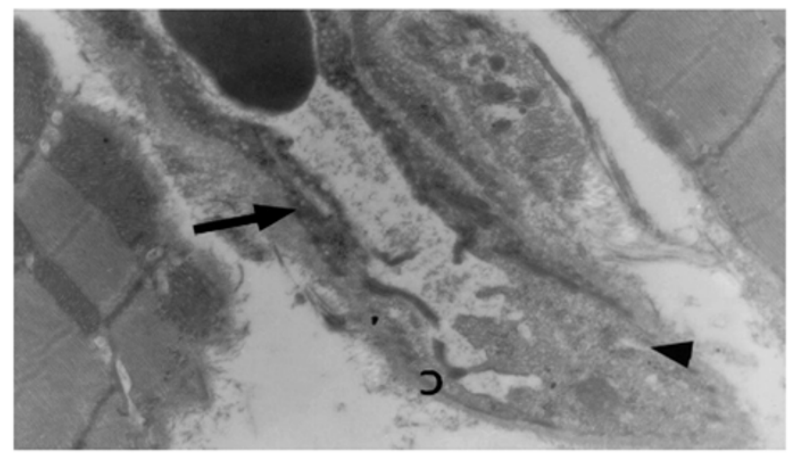

Fig. 3 Cytochemical detection of capillary NO synthase reaction product in capillary endothelial cells revealed cells with (arrow) as well as without (arrow head) specific reaction product. Magnification: $\times 20,000$.

geneous - reversible till irreversible subcellular endothelial cell alterations disseminated in hearts of both experimental groups [Fig. 4A-D]. Thickened basal lamina was prominent feature of diabetic heart capillaries, while in L-NAMEtreated rats thickening was less considerable. Oedematous endothelial cells contained a low number of pinocytic vesicles, ribosomes, electrolucent nucleus, partially swollen mitochondria and/or ones with ruptured cristae. Intercellular junctions of endothelial cells were injured, too. Some cells revealed various density of cytoplasm. Capillary lumen was often narrowed and/or irregular due to extensive protrusions and bridging of endothelium into lumen. These alterations indicating cell injury were more significant in the myocardium of diabetic animals.

Beside of structural markers of capillary injury, in the hearts of both groups there were also found ultrastructural markers of micro-angiogenesis - process of formation of new capillaries [Fig. 5A-D]. They were manifested by alterations of endothelium and shape of pericytes which during normal conditions closely line capillary. Close contact between endothelial cell and pericyte became gradually lost due to frequent interaction pericyte-fibroblast in flocculent thickened basal lamina, elongated shape of pericyte was shortened and it appeared to peel of capillary. Migration of endothelial cells, unusually small newly formed capillaries with a small lumen and enriched with ribosomes and pinocytic vesicles were observed as further subcellular markers of angiogenesis. To compensate oxygen supply cardiomyocytes themselves formed capillary-like structures. Subcellular angiogenic markers were found to be more frequently in hypertensive rats.

\section{DISCUSSION}

Functional and structural cardiac impairment in animals 

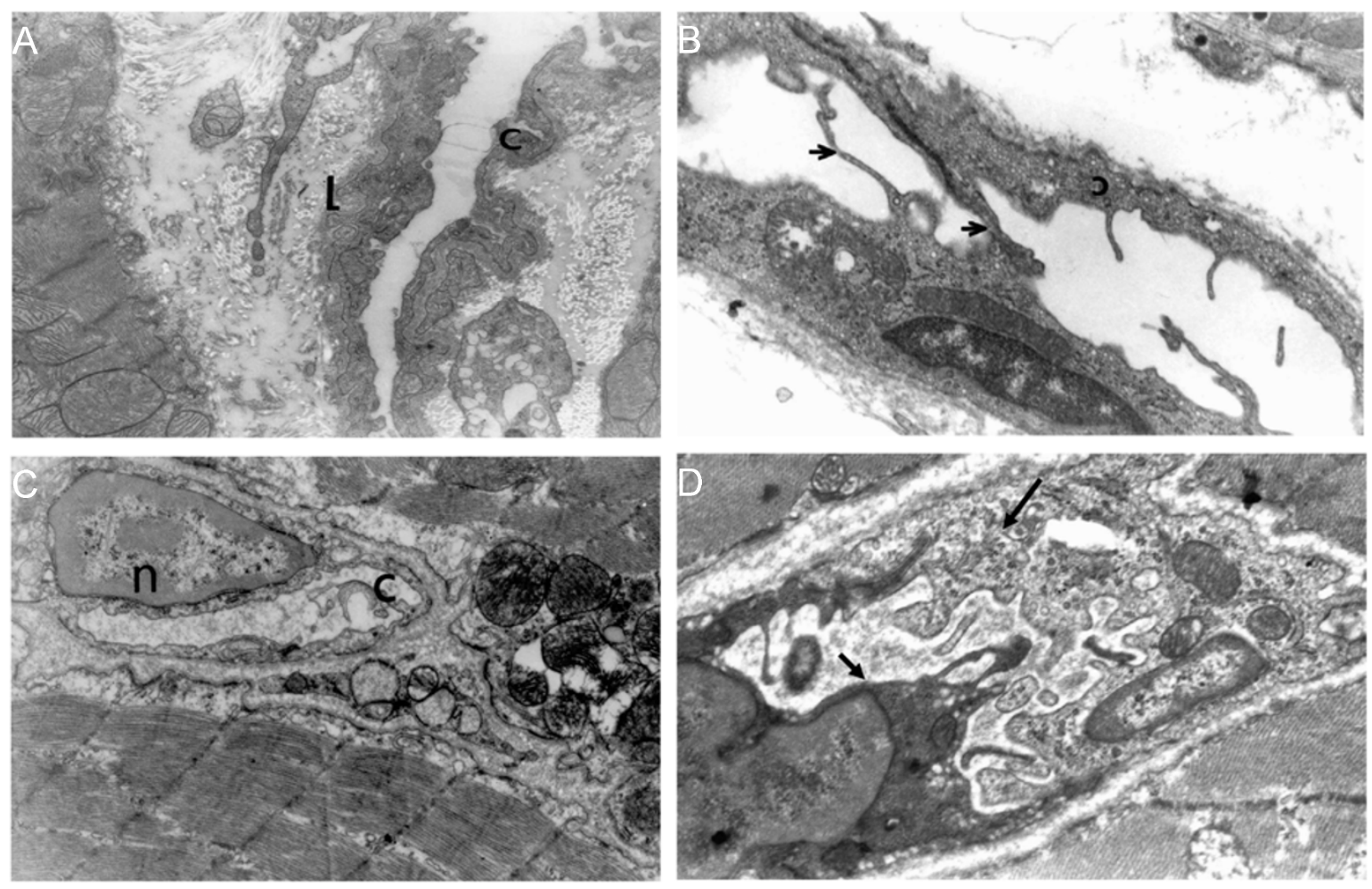

Fig. 4 Subcellular injury of cardiac capillaries in diabetic (A, B) and hypertensive (C, D) rats demonstrated by (A) - thickened basal lamina (L), (B) - endothelial bridging and protrusions (arrows), (C) - oedematous endothelial cell (c) containing electrolucen nucleus (n), and (D) - nonuniform injury of endothelial cells in one capillary characterised by various density of cytoplasm (arrows). Magnification: A $\times 13,000 ; \mathbf{B} \times 15,000 ; \mathbf{C} \times 24,000 ; \mathbf{D} \times 26,000$.

with chronic diabetes induced by STZ or sustained hypertension due to long-term application of L-NAME have been documented in many studies. Associated with the myocardial dysfunction, severe anatomical alterations insufficient growth and rarefaction - were found in the terminal exchange vessels - capillaries [13, 15, 23]. These changes are closely followed by endothelial dysfunction which is due to chronic accumulation of glucose in diabetes, while in hypertensive animals it results from the inhibition of $\mathrm{NO}$ generation with $\mathrm{NO}$ synthase inhibitor LNAME. The endothelial dysfunction is associated with the imbalance of secreting vasoactive mediators including $\mathrm{NO}$ $[12,24]$. NO modulates various physiological activities such as homeostasis, vascular tone, defensive reactions [25], and its decreased bioavailability contributes to impairment of NO-dependent processes in the myocardium. Reduced NO bioactivity may results from lower NO production [e.g. the inhibition of NOS activity, a deficit of Larginine as the natural NO donor [26]] or via enhanced NO degradation [increased oxidative stress and generation of oxygen free radicals [27, 28]] contributed to the impairment of NO-dependent mechanisms. Light micros- copy in our studies $[10,29]$ correlates with quantitative image analysis showing significantly low capillary NOS activity within the myocardial tissue of both groups indicating diminished production of $\mathrm{NO}$ and the endothelial dysfunction. Rare focal micro-areas with persisting activity of NOS, which has also seen on electron-microscopic level, stress different vulnerability of endothelial cells to pathophysiological state. Local areas with decreased/abolished activity of alkaline phosphatase in arterial part of capillary bed also supported the endothelial dysfunction and/or capillary reduced density minimising the transport of oxygen and substances to neighbouring cardiomyocytes. In contrast, DPP IV activity as the enzyme marker of venular capillaries was not apparently changed by diabetes or hypertension. Our quantitative data correlated with qualitative ones demonstrated that the reaction density of all detected enzymes - AlPh, DPP IV and NOS - was lower in diabetic animals than in hypertensive rats. As it is known diabetes is multifactorial disease and hyperglycaemia itself has deleterious effect on endothelial cell function [15]. In addition, hyperglycaemia promotes the cascade of further signalling and metabolic abnormalities e.g. increased pro- 

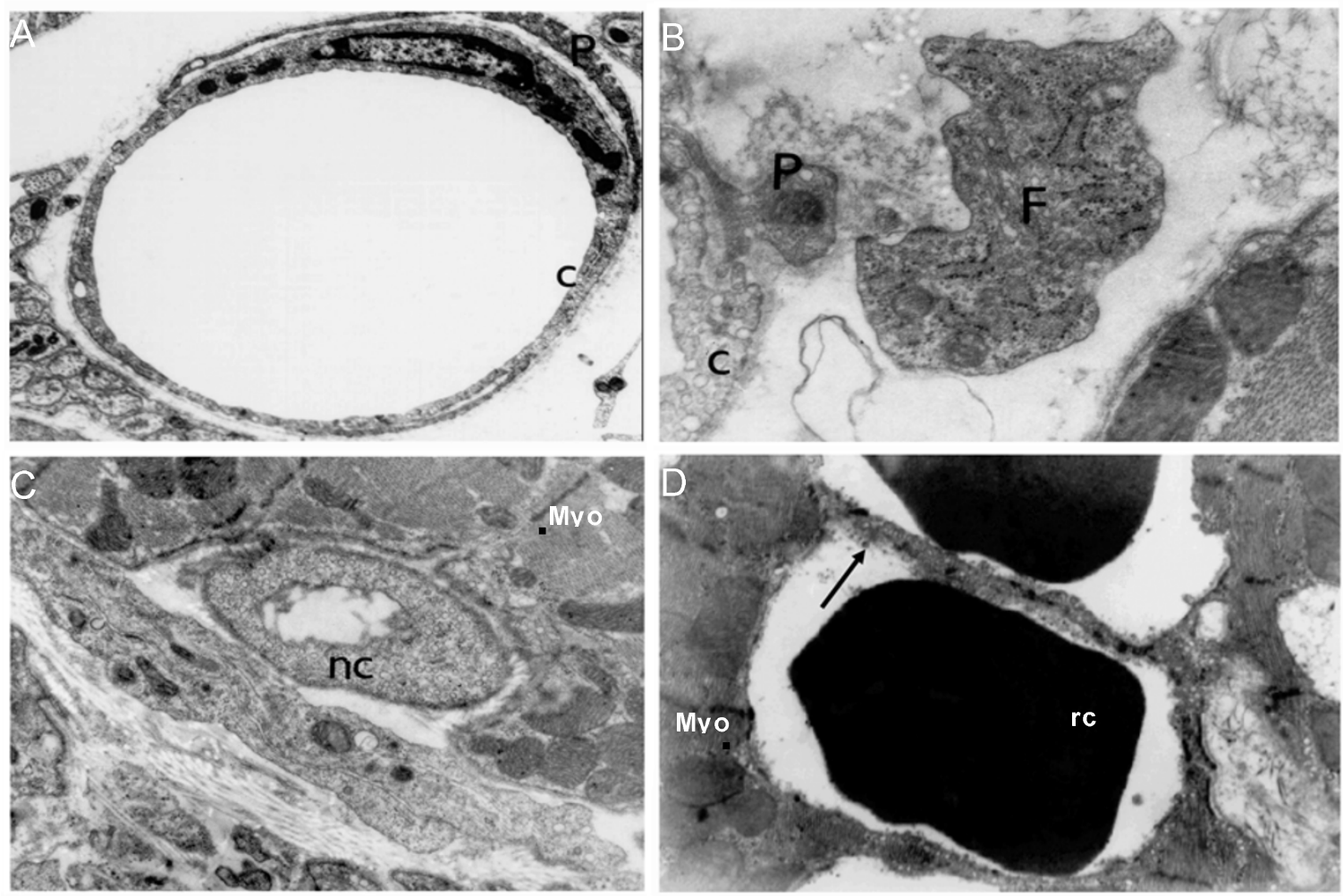

Fig. 5 Ultrastructural markers of angiogenesis were seen in the myocardium of hypertensive and diabetic rats. (A) - normal appearance of capillary (c) covered by pericyte (P). (B) - prolongation of pericyte $(\mathrm{P})$ shape and its interaction with fibroblast (F). (C) - new small capillary (nc) containing high number of pinocytic vesicles and narrow lumen. (D) - capillary-like structure (arrow) formed by cardiomyocyte (Myo). rd - red cell. Magnification: A ×15, 000; $\mathbf{B} \times 30,000 ; \mathbf{C} \times 28,000 ; \mathbf{D} \times 33,000$.

duction of oxygen free radicals and reduced antioxidative defence [31, 32], defects in intracellular Ca handling [33] which may have additional destructive effects on the function of capillary endothelium. NO-deficient hypertension is also accompanied by various metabolic disturbances, including enhanced production of oxygen free radicals [34], reduced activity of Na, K-ATPase [35], and increased lactate dehydrogenase activity [9] which may contribute to endothelial injury. However, it dosn't only cause such deleterious effects. Histochemical techniques used in our previous studies have shown heterogeneously reduced activities of some other enzymes involved in metabolic and energetic processes in the heart of diabetic and hypertensive rats $[8,11,30]$ that may contribute to its dysfunction.

In addition to enzyme histochemistry, our studies demonstrated that capillary endothelial cells revealed similar reversible and irreversible subcellular alterations in the myocardium of diabetic and hypertensive animals $[8,11$, $30,37]$ manifested by thickening of basal membrane, diminished number of ribosomes and pinocytic vesicles, injured mitochondria and electrolucent nuclear chromatin, various density of cytoplasm together with endothelial cell swelling accompanied by luminal narrowing, diversity of capillary calibre, endothelial extravastion, branching of capillaries and pericapillary fibrosis. They represent structural form of endothelial dysfunction and seem to make up an integral part of degradation processes in capillaries and might contribute to accelerated disappearance of capillary endothelium. Comparing diabetic and hypertensive animals, these abnormalities were more frequent in diabetic and correlate with histochemistry

In response to stressors, capillaries represent a critical element not only in the angiopathy. Capillary endothelial cells also play a significant role in the process by which capillaries proliferate and differentiate from pre-existing ones - angiogenesis $[38,39]$ and which develops as one of compensatory mechanisms in the failing heart. Angiogenesis consists of a several distinct steps - activation of endothelium, degradation of basal membrane, proliferating and migrating, re-differentiation and recruitment of pericytes along the newly formed capillary structure. Mentioned ultrastructural markers were found in the myocardium of both diabetic and hypertensive rats $[30,40]$. However, they were more evident in hypertensive animals. 
The initiation of angiogenesis depends on a dynamic balance between numerous pro- and anti-angiogenic factors in the immediate environment of endothelial cells as well as in extracellular matrix [39]. The NO is one of the most important mediator of angiogenesis. However, in the literature there exists controversial data supporting its pro-angiogenic [41] and its anti-angiogenic effects as well [42]. Our results showing reduced endothelial NOS activity and subcellular markers of angiogenesis in both experimental groups support anti-angiogenic effect of NO. Diabetes and hypertension are characterized by reduced NO bioavailability and hypoxia. Hypoxia is known to upregulate transcription factor HIF 1 activating expression of genes involved in angiogenesis [43]. However, it was demonstrated that co-existence of both hypoxia and lower NO concentration caused a rapid decrease in HIF1 $[44,45]$ that may represent one of the mechanisms involved in anti-angiogenic efect of NO.

In conclusion, our results indicate that two different models of heart failure the STZ-induced diabetes and NOdeficient hypertension were accompanied by similar structural abnormalities of capillary endothelial cells that suggest to endothelial angiopathy as well as endothelial angiogenesis.

\section{ACKNOWLEDGEMENTS}

This work was supported by VEGA grant agency, grants No. $2 / 5021 / 25,2 / 5009 / 25$ and $2 / 3124 / 23$. We thank A. BRICHTOVA, A. MACSALIOVA, and T. OTTOVA for excellence technical assistance.

Received, Oct 18, 2004

Revised, May 15, 2005

Accepted, July 14, 2005

\section{REFERENCES}

1 Ribeiro MO, Antunes E, De-Nucci G, Lovisolo SM, Zats R. Chronic inhibition of nitric oxide synthesis. A new model of arterial hypertension. Hypertension 1992; 20:298-303.

2 Pechánová $\mathrm{O}$, Bábal $\mathrm{P}$, Bernátová I. Pathological changes in rat heart after long-term nitric oxide synthase inhibition. Endothelium 1995; 3:93-8.

3 Ravingerová T, Styk J, Pancza D, et al. Diabetes cardiomyopathy in rats: allevation of myocardial dysfunction caused by $\mathrm{Ca}^{2+}$ overload. Diabetes Res Clin Pract 1996; 31 Suppl:S105-12.

4 Ziegelhoffer A, Ravingerova T, Styk J, et al. Diabetic cardiomyopathy in rats: biochemical mechanisms of increased tolerance to calcium overload. Diabetes Res Clin Pract 1996; 31 Suppl: S93-103.

5 Koltai MZS, Balogh I, Wagner M, Pogatsa G. Diabetic myocardial alterations in ultrastructure and function. Exp Path 1984; 25:215-21.

6 Factor SM, Borczuk A, Charron MJ, et al. Myocardial alterations in diabetes and hypertension. Diabetes Res Clin Pract
1996; 31 Suppl:S133-42.

7 Kristek F, Gerova M, Devat L, Varga I. Remodelling of septal branch of coronary artery and carotid artery in L-NAME treated rats. Physiol Res 1996; 45:329-33.

8 Tribulova N, Ravingerova T, Volkovova K, et al. Resistance of diabetic rat hearts to $\mathrm{Ca}$ overload-related injury. Histochemical and ultrastructural study. Diabetes Res Clin Pract 1996; 31 Suppl: S113-22.

9 Babal P, Pechanova O, Bernatova I, Stvrtina S. Chronic inhibition of NO synthesis produces myocardial fibrosis and arterial media hyperplasia. Histol Histopathol 1997; 12:623-9.

10 Pechanova O, Bernatova I, Pelouch V, Babal P. Protein remodelling of the heart in NO-deficient hypertension: the effect of captopril. J Mol Cell Cardiol 1997; 29:3365-74

11 Tribulova N, Okruhlicova L, Bernatova I, Pechanova O. Chronic disturbances in NO production results in histochemical and subcellular alterations of the rat heart. Physiol Res 2000; 49:77-88.

12 Tooke JE, Morris SJ, Shore AC. Microvascular functional abnormalities in diabetes: the role of the endothelium. Diabetes Res Clin Pract 1996; 31 Suppl:S127-32.

13 Pries AR. Structural autoregulation of terminal vascular beds: vascular adaptation and development of hypertension. Hypertension 1999; 33:153-61.

14 Park JB. Small artery remodelling is the most prevalent (earliest?) form of target organ damage in mild essential hypertension. J Hypertens 2001; 19:921-30.

15 Reusch JEB. Diabetes, microvascular complications, and cardiovascular complications: what is it about glucose? J Clin Invest 2003; 112:986-8.

16 Hirschi KK, D’Amore PA. Pericytes in the microvasculature. Cardiovasc Res 1996; 32:687-98.

17 Vapaatalo H, Mervaala E. Clinically important factors influencing endothelial function. Med Sci Monit 2001, 7:1075-85.

18 Cines DB, Pollak ES, Buck CA, et al. Endothelial cells in physiology and in the pathophysiology of vascular disorders. Blood 1998; 91:3527-61.

19 McCarthy SA, Kuzu I, Gatter KC, Bicknell R. Heterogeneity of the endothelial cells and its role in organ preference of tumour metastasis. TIPS 1991; 12:462-79.

20 Broulan JP, Gilbert MA, Bonneau M, et al. Macro and microheterogeneity in normal endothelial cells: Differential composition of luminal glycocalyx and functional implications. Endothelium 1999; 6:251-62.

21 Klimaschewski L, Kummer W, Mayer B, et al. Nitric oxide synthase in cardiac nerve fibres and neurons of rat and guinea pig heart. Circ Res 1992; 71:1533-7.

22 Lojda Z, Gossrau R, Schiebler TH, eds. Enzym-Histochemische Methoden. Berlin: Sringer-Verlag, 1976.

23 Boudier HA. Hypertension and microcirculation. Arch Mal Coeur Vaiss 2002; 95:17-22.

24 Kiowski W. Endothelial dysfunction in hypertension. Clin Exp Hypertens 1999; 21:635-46.

25 Kubes P, Suzuki M, Granger DN. Nitric oxide: an endogeneous modulator of leukocyte-adhesion. Proc Natl Acad Sci USA 1991; 88:4651-5.

26 Pieper GM, Peltier BA. Amelioration by L-arginine of a dysfunctional arginine/nitric oxide pathway in diabetic endothelium. J Cardiovasc Pharmacol 1995; 25:397-403.

27 Rubbo H, Radi R, Trujillo M, et al. Nitric oxide regulation of 
superoxide and peroxynitrite-dependent lipid peroxidation: Formation of novel nitrogen-containing oxidized lipid derivates. J Biol Chem 1994; 269:26066-75.

28 Oberley LW. Free radicals and diabetes. Free Radic Biol Med 1998; 5:113-24.

29 Okruhlicova L, Tribulova N, Macsaliova A, et al. Endothelial dysfunction is associated with both, angiopathy and angiogenesis. J Mol Cell Cardiol 2001; 33:A87.

30 Okruhlicova L, Sotnikova R, Stefek M, et al. L-Arginine reduces structural remodeling in the diabetic rat myocardium. Methods Find Exp Clin Pharmacol 2002; 24:201-7.

31 Van Dam PS, Bravenboer B. Oxidative stress and antioxidant treatment in diabetic neuropathy. Neurosci Res Commun 1997; 21:41-8.

32 Baynes JW. Role of oxidative stress in development of complications in diabetes. Diabetes 1991; 40:405-12.

33 Makino A, Dhalla NS, Elimban V. Sarcolemmal $\mathrm{Ca}^{2+}$ transport in streptozotocin-induced diabetic cardiomyopathy in rats. Am. J. Physiol 1987; 253:E202-7.

34 Ziacikova J, Pechanova O, Bernatova I, Vrbjar N. Cardiac membrane proteins and phospholipids in L-NAME induced hypertension. Gen Physiol Biophys 1999; 18:13-5.

35 Javorkova V, Pechanova O, Andriantsitohaina R, Vrbjar N. Effect of polyphenolic compounds on the renal $\mathrm{Na}, \mathrm{K}$-ATPase during development and persistence of hypertension in rats. Exp. Physiol 2003; 89:73-81.

36 Johns S. Impaired endothelial function in arterial hypertension and hypercholesterolemia: potential mechanisms and differences. J Hypertens 2000; 18:363-74.
37 Stefek M, Sotnikova R, Okruhlicova L, et al. Effect of dietary supplementation with the pyridoindole antioxidant stobadine on antioxidant state and ultrastructure of diabetic rat myocardium. Acta Diabetol 2000; 37:111-7.

38 Rakusan K. Vascularization of the heart during normal and pathological growth. Adv Organ Biol 1999; 7:129-53.

39 Kutryk MBJ, Steward DJ. Angiogenesis of the heart. Microsc Res Tech 2003; 60:138-58.

40 Okruhlicova L, Tribulova N, Bernatova I, Pechanova O. Induction of angiogenesis in NO-deficient rat heart. Physiol Res 2000; 49:71-9.

41 Zhao X, Xiangru L, Qingping F. Deficiency in endothelial nitric oxide synthase impairs myocardial angiogenesis. Am J Physiol 2002; 283:H2371-8

42 Pipili-Synetos E, Sakkoula E, Haralobopoulos G, et al. Evidence that nitric oxide is an endogeneous antiangiogenic mediator. $\mathrm{Br} \mathrm{J}$ Pharmacol 1994; 11:894-902.

43 Semenza GL. Signal transduction to hypoxia-inducible factor. Biochem Pharmacol 2002; 64:993-8.

44 Mateo J, Garcia-Lecea M, Cadenas S, et al. Regulation of hypoxia-inducible factor-1alpha by nitric oxide through mitochondria-dependent and independent pathways. Biochem J 2003; 376:537-44.

$45 \mathrm{Xu} \mathrm{W}$, Charles IG, Moncada S. Nitric oxide: orchestrating hypoxia regulation through mitochondrial respiration and the endoplasmic reticulum stress response. Cell Res 2005; 15:63-5.

Editor: Dr. Wei Ming XU 